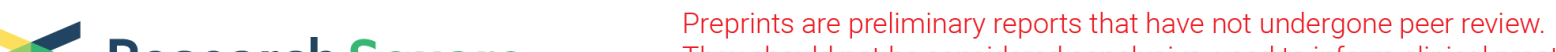 Research Square They should not be considered conclusive, used to inform clinical practice, or referenced by the media as validated information.
}

\section{The ERG1 Potassium Channel is More Abundant in Skeletal Muscle from Cachectic than Healthy Humans}

\section{Sandra Zampieri}

University of Padua

Marco Sandri

University of Padua

Joseph L. Cheatwood

Southern Illinois University

Rajesh P. Balaraman

Sam Houston State University College of Science \& Engineering Technology

\section{Luke B. Anderson}

Southern Illinois University Carbondale

\section{Brittan A. Cobb}

Southern Illinois University Carbondale

Chase D. Latour

University of North Carolina at Chapel Hill

Gregory H. Hockerman

Purdue University

\section{Helmut Kern}

Phyisko- und Rheumatherapie $\mathrm{GmbH}$

\section{Roberta Sartori}

Universita degli Studi di Padova

\section{Barbara Ravara}

Universita degli Studi di Padova

\section{Stefano Merigliano}

Universita degli Studi di Padova Scuola di Medicina e Chirurgia

\section{Gianfranco Da Dalt}

Universita degli Studi di Padova Scuola di Medicina e Chirurgia

\section{Judith K. Davie}

Southern Illinois University Carbondale

\section{Punit Kohli}

Southern Illinois University Carbondale

\section{Ugo Carraro}


Universita degli Studi di Padova

Amber L Pond ( $\boldsymbol{\sim}$ apond@siumed.edu )

Southern Illinois University https://orcid.org/0000-0002-2836-889X

\section{Research}

Keywords: ether-a-go-go related K+ channel, dystrophin, dihydropyridine receptor, t-tubules, sarcolemmal membrane, skeletal muscle

Posted Date: March 17th, 2020

DOI: https://doi.org/10.21203/rs.3.rs-17510/v1

License: (c) (i) This work is licensed under a Creative Commons Attribution 4.0 International License. Read Full License 


\section{Abstract}

Background: The ERG1a potassium channel has been detected in the atrophying skeletal muscle of mice experiencing either muscle disuse or cancer cachexia and further evidenced to contribute to muscle deterioration by enhancing ubiquitin proteolysis; however, to our knowledge, ERG1 has not been reported in human skeletal muscle.

Methods and Results: Here, using immunohistochemistry, we detect ERG1 immunofluorescence in human Rectus abdominis skeletal muscle sarcolemma. Further, using single point brightness data, we report detection of ERG1 immunofluorescence at low levels in the Rectus abdominis muscle sarcolemma of young adult humans and show that it trends toward greater levels (10.6\%) in healthy aged adults. Interestingly, we detect ERG1 immunofluorescence at a statistically greater level $(53.6 \% ; p<0.05)$ in the skeletal muscle of older people having cancer cachexia than in age-matched adults. Importantly, using immunoblot, we reveal that ERG1 protein is $38 \%(p<0.09)$ more abundant in the skeletal muscle of cachectic older adults than in healthy age-matched controls. Additionally, we report that the ERG1 fluorescent pattern is consistent with I-band localization.

Conclusions: The data suggest that ERG1 may be related to muscle loss in humans and is located in ttubules where it could influence calcium handling.

\section{Background}

Skeletal muscle atrophy is defined as a decrease in muscle contractile proteins that implies a weakening of strength. Atrophy occurs with normal aging and can also result from muscle disuse as well as muscle and/or neural damage or disease [1-4]. Age-related skeletal muscle atrophy (i.e., sarcopenia) has significant negative impacts on the health and quality of life for older persons [5]. The wasting syndrome (i.e., cachexia) that often affects cancer patients and other critically ill people (e.g., AIDS, sepsis, etc.) includes severe skeletal muscle atrophy and contributes to human morbidity and mortality [6-9]. Exercise is purported to be the most beneficial treatment for muscle atrophy [10-12]; however, many injured or ill persons are unable to participate adequately in this therapy. Additional strategies being explored to combat atrophy include nutritional therapy $[2,10]$ and administration of pharmaceuticals such as proteolysis inhibitors [13], growth factors [14], beta-agonists [3], protein synthesis stimulators [15], and myostatin inhibitors [16]. Each has minimal efficacy and/or drawbacks, necessitating the development of more effective treatments. Therefore, an improved understanding of the mechanism(s) modulating atrophy is essential.

The ether-a-go-go-related gene (ERG1) is a voltage-gated potassium channel known to contribute to repolarization of the cardiac action potential in numerous mammalian species [17]. Alternative splice variants of ERG1 (ERG1A and 1B) have been identified in both mice (mouse ERG1, i.e., MERG1a and MERG1b [18]) and humans (human ERG1, i.e., HERG1a and HERG1b [19]). HERG1a and MERG1a are highly homologous full length isoforms with 1159- and 1162-amino acids, respectively; MERG1a is $96 \%$ 
identical to HERG1a [18]. HERG1b and its mouse homolog MERG1b both have an alternate N-terminus which lacks the first 342 amino acids of the ERG1a variant, having instead a different 36 amino acid sequence; ERG1b is otherwise basically identical to ERG1a in terms of primary structure [18,19]. ERG1 gene expression and the ERG1 protein have been reported in numerous tissues of various species [20, 21]; however, ERG1 detection is most prevalently reported and researched in the mammalian heart [17-19, 21-23] where the ERG1 channel has been shown to be a hetero-multimer of both ERG1a and 1b [23].

Previously, we reported detection of the MERG1a isoform protein and Merg1a mRNA in skeletal muscle atrophied as a result of disuse (unweighting) and cancer cachexia, with detection of ERG1a mRNA and protein abundances being negligible in healthy skeletal muscle [24], as reported earlier by others [18]. Further, our studies have shown that expression of wild type (WT) Merg1a in the Gastrocnemius muscle of weight bearing mice induces atrophy and that ectopic expression of a dominant negative Merg1a mutant (DN-Merg1a [25]) suppresses atrophy in unweighted mice. Importantly, we have shown that ectopic expression of Merg1a in healthy mouse skeletal muscle increases abundance of the skeletal muscle-specific ubiquitin proteasome pathway (UPP) E3 ligase, MuRF1, and up-regulates UPP activity [24, 26 , 27]. Here, using immunohistochemistry $(\mathrm{IHC})$, we show for the first time to our knowledge, that the ERG1 channel is detected in human Rectus abdominis (RA) muscle and that it is significantly more abundant in the RA of humans experiencing cachexia than in RA from both age-matched healthy control and healthy young adults. Additionally, using immunoblot we show that ERG1 is more abundant in leg muscle samples taken (during above-knee amputations) from cachectic people than in those with a normal body mass index. Further, similar to the heart, we demonstrate in skeletal muscle that ERG1 immunofluorescence is localized in the sarcolemma and is consistent with a t-tubule distribution.

\section{Methods}

Human Skeletal Muscle.

Muscle biopsies used for the immunohistochemistry were from healthy adult humans aged either 2429 years $(n=4)$ or $61-86$ years $(n=7)$ and from cachectic patients aged $65-88$ years $(n=5$; Table 1 , Figs. 1 and 2). Samples were obtained during elective surgery by cold section of Rectus abdominus muscle. Healthy subjects were control donors who were undergoing elective laparotomy for nonneoplastic and non-inflammatory diseases. Cachectic patients affected with colorectal, esophago-gastric or pancreatic cancers were enrolled for a project approved by the Ethical Committee for Clinical Experimentation of Padova (protocol number 3674/AO/15). Cachexia was diagnosed according to criteria described by Fearon et. al., (2011; [28]). All biopsies were immediately frozen in pre-chilled isopentane and stored in liquid nitrogen until use. All enrolled subjects were volunteers who signed written informed consent forms. Muscle biopsies were de-identified before use. The skeletal muscle samples from limbs amputated just above the knees of patients with either a normal $(>24)$ or low $(<18)$ BMI ( $n=6$; Table 2, Fig. 3A-C) were purchased from The Collaborative Human Tissue Network (CHTN), Midwestern Division. The human Rectus abdominis muscle (RA) samples and the malignant esophageal 
muscle sample used for the immunoblot ( $n=4$; Table 2, Fig. 3D, E) were provided by the SIU School of Medicine Tissue Bank, a Simmons Cancer Institute funded program.

Rat Skeletal Muscle. The FBN rat (National Institute of Aging Rodent Service) muscles used for the immunohistochemistry were generous gifts from Dr. Don Caspary (Southern Illinois University School of Medicine; Springfield, IL). Dr. Caspary's animal studies are all approved by the SIU School of Medicine Institutional Animal Care and Use Committee.

Antibodies. For immunohistochemistry, the ERG1 antibodies (P9497 and AB5908) were purchased from Sigma (St. Louis, MO) and diluted (see IHC methods below) prior to use. Similar results were obtained with each antibody, although the P9497 appeared to have a higher affinity for the ERG1 protein. The dystrophin (MAB1645MI) and dihydropyridine receptor (DHPR; MAB-920) antibodies were purchased from Thermo-Fisher Scientific (Waltham, MA) and diluted 1:20 and 1:100, respectively prior to use. The MHC antibody (MF20 [29]) was purchased from the Developmental Studies Hybridoma Bank (DSHB; lowa City, IA) and diluted 1:10. The a-actinin antibody (MA1104) was purchased from Boster Biological Technology (Pleasanton, CA) and diluted to $2 \propto \mathrm{g} / \mathrm{mL}$ before use. The fluorescent secondary antibodies (goat anti-rabbit $\lg G$ antibody Alexa Fluor 488 and goat anti-mouse $\lg G$ antibody Alexa Fluor 568) were also purchased from ThermoFisher. The Tyramide SuperBoost Kit with Alexa Fluor 488 (ThermoFisher) was used to enhance ERG1 protein signal when muscle was co-immuno-stained for ERG1 and both myosin heavy chain and a-actinin. For immunoblot of human muscle we used an "in house" ERG1 antibody [30] and stained the membrane with Coomassie Blue once we had imaged the chemiluminscence (Sigma; St. Louis, MO). The alkaline phosphatase conjugated goat anti-rabbit IgG secondary antibody was purchased from Sigma.

Immunohistochemistry. Tissue samples were cryo-sectioned $(20 \propto \mathrm{m})$ and fixed in methanol at $-20{ }^{\circ} \mathrm{C}$ for 10 minutes. These were then rinsed with PBS at room temperature (RT) and incubated in $0.3 \% \mathrm{H}_{2} \mathrm{O}_{2}$ for 5 minutes (except those for which the Tyramide kit was used) followed by rinse in RT PBS. Sections used with the Tyramide kit (those co-immunostained for myosin and ERG1 and also for a-actinin and ERG1) were incubated with $3 \% \mathrm{H}_{2} \mathrm{O}_{2}$ for 1 hour and then rinsed with PBS. All sections were then dipped in filtered $0.3 \%$ Sudan Black in $70 \%$ ethanol to quench auto-fluorescence. These were then rinsed in PBS. At this point, the rat muscle sections stained using the Tyramide SuperBoost kit were treated according to the manufacturer's instructions with antibody incubation times described as follows for the remaining sections. All other sections were incubated with blocking reagent I (10\% normal goat serum [NGS; Sigma, St. Louis, MO], 0.1\% bovine serum albumin [BSA; Sigma] and 0.1\% Tween-20 in PBS) for one hour at RT. These slides were then incubated overnight at $4{ }^{\circ} \mathrm{C}$ in either primary antibody diluted in blocking reagent II (PBS with $5 \%$ NGS, $0.05 \%$ BSA, $0.1 \%$ Tween- 20 and $0.1 \%$ sodium azide) or in blocking reagent II only as a control for primary antibody binding. The sections were rinsed in PBS containing $0.1 \%$ Tween-20 and incubated for 1 hour at RT in a second primary antibody (where dual staining was desired) and then rinsed again in PBS containing $0.1 \%$ Tween-20. Sections were incubated in secondary antibody (as above) diluted 1:1000 with blocking reagent II. Finally, all sections were rinsed with PBS and mounted with Fluoromount G containing DAPI (Electron Microscopy Sciences; Hatfield, PA). 
Imaging. Images in Figs. 1 and 2 were acquired using a Leica DM4500 microscope with a Leica DFC 340FX camera. Images in Figs. 4 and 5 were acquired using a Leica TCS SP5 II Spectral Confocal System with a HCX PL APO CS 63X/1.40-0.60 oil objective. Argon (488 nm) and HeNe (543 nm) lasers were used to excite fluorophores of myosin and ERG1. The cross talk was prevented by PMTs detection window adjustments with emission detector band width of $30 \mathrm{~nm}$ and improved SNR was achieved through increased line and frame averaging $(n=6)$ with pinhole of 1 AU (LAS X software). Acquisition parameters for all sections were maintained identically across all samples allowing reliable comparison of immunofluorescence intensities for different samples. Images in Fig. 6 were acquired using a Leica SPE Confocal microscope with an ACS APO 63x/1.30 oil objective.

Quantitative Analysis and Statistics. Human Samples. For the immunohistochemistry in Fig. 2, treatment groups were: 1) young healthy adults; 2) elder healthy adults; and 3) elder cachectic adults (Table 1). Images of two sections per individual human were evaluated by two independent observers who were blind to treatment groups. For each tissue section, three fields were imaged and the single point brightness was measured for 50 random consecutive points within the sarcolemma of each complete fiber within each field using ImageJ [31] with methods adapted from those published previously [32]. Brightness values were recorded as integers ranging from 0 (no signal) to 256 (white) and the average brightness value ( \pm standard error of the mean, SEM) for each section was determined arithmetically. The resulting data were normalized to the young adult sample average and analyzed by two-way ANOVA using the General Linear Model Procedure of SAS 9.4 (SAS Institute Inc., Cary, NC), with group and observer as factors in the model. First, we tested significance of the interaction between group and observer. Because the interaction was non-significant, the term was removed and the data from the two observers were combined per sample to represent a single replicate. All subsequent analyses were completed using a two-way ANOVA without the interaction term. Means were compared between groups using Tukey's simultaneous confidence intervals to adjust for multiple comparisons. The muscle fiber diameter data was analyzed by a one-way ANOVA using the General Linear Model Procedure of SAS 9.4 (SAS Institute, Inc.) and the means were compared using Tukey's simultaneous confidence intervals as described for the brightness data above. For power analysis we used the Power Procedure of SAS 9.4 (SAS Institute, Inc.). For Fig. 3, Optical Density values of muscle sample proteins in the immunoblot were determined using ImageJ [31] and compared using a Student's t-test. Analyses were considered significant for $p<0.05$. Rat Samples. Pearson correlation analyses were performed using GraphPad Prism version 7.02 for Windows (GraphPad Software, La Jolla California USA).

Immunoblot. Membrane proteins were extracted from human skeletal muscles [33] and the protein content of each homogenate was measured using a DC Protein Assay Kit (Bio-Rad; Hercules, CA). Aliquots of equal protein content were immunoblotted as described earlier [24]. Briefly, muscle homogenate samples (50 $\propto$ g protein) were electrophoresed through a polyacrylamide gel (4-20\% gradient), transferred to PVDF membrane (BioRad; Hercules, CA) and immunoblotted using an "in house" ERG1 antibody [30] and an Immun-Star Western Chemiluminescent Kit (Bio-Rad). Finally, the blotted membrane was stained with Coomassie Blue to confirm that membrane samples contained equal protein. 


\section{Results}

ERG1 Protein Localizes to the Sarcolemmal Membrane of Human Skeletal Muscle. To explore ERG1 localization, human Rectus abdominis (RA) serial sections were immuno-stained without primary antibody as controls (Fig. 1A-D). The absence of green or red fluorescence in the control sections (Fig. 1A) demonstrated that any red or green fluorescence in the other images is a product of primary antibody binding. Serial RA sections were also concurrently co-immuno-stained for ERG1 and the sarcolemmal membrane marker dystrophin. We detected dystrophin fluorescence (red) in the sarcolemmal membrane as expected (Fig. 1B). Fluorescent confocal imaging also detected ERG1 fluorescence (green) in the sarcolemmal membrane of human RA muscle (Fig. 1C). The appearance of a mild mottled green fluorescence pattern within the cytoplasm is consistent with localization also occurring within the cell. Merged images of the sections demonstrate that dystrophin and ERG1 both localize to the sarcolemmal membrane of human RA, although the absence of consistent yellow color from the merged fluorescent images suggests that the proteins do not co-localize in the same pattern (Fig. 1D). Further, localization of both of these proteins to the sarcolemma is supported by merged images from which ERG1 and dystrophin fluorescence intensity profile measurements are discovered to follow a very similar pattern (Fig. 1E) through the demarcated (white rectangle, Fig. 1D) region of the sarcolemma. The Pearson correlation statistic of the profile data (red compared to green fluorescence intensitites) for this single representative image was 0.96 , demonstrating that the two fluorescent signals were similarly localized within the sarcolemmal membrane. The patterns noted here were similar for all human samples evaluated ( $\mathrm{n}=3$ cachectic samples). The blue structures are DAPI stained nuclei. ERG1 and dystrophin proteins are also both similarly located in rat muscle sarcolemma (data not shown).

The ERG1 Channel Protein is Detected in Human Rectus Abdominis (RA) Muscle at Greater Abundance in Cachectic than in Age-Matched Healthy Tissue. Human RA muscle samples were cryo-sectioned and stained with hematoxylin and eosin (Fig. 2A-C). The morphology of the muscle from the healthy adults (both younger and aged) is similar, having an expected normal structure and average myofiber diameter: 1) healthy young people $=52.8 \pm 7.8 \propto \mathrm{m}$, and 2) healthy seniors $=53.6 \pm 10.5 \propto \mathrm{m}$. Indeed, there was no significant difference in size of the fibers from the healthy people. However, fiber sections from the cachectic seniors displayed fiber polymorphism with a smaller average fiber diameter, $39.0 \pm 7.9 \propto \mathrm{m}$, being $27.2 \%$ smaller than those of the healthy age-matched controls and $26.1 \%$ smaller $(p<0.05)$ than those of the healthy young persons. Serial sections were further evaluated by immuno-stain procedures without primary antibody to serve as control (Fig. 2D). The absence of fluorescence in control sections demonstrates that any red or green fluorescence in the other sections is a product of primary antibody binding. Sections of RA from young healthy people and from older healthy and age-matched (older) cachectic people were immuno-stained for ERG1 (Fig. 2E-G). Indeed, ERG1 fluorescence was detected in the sarcolemma of all samples. (Note also the mottled green appearance of the fiber interiors, suggesting that ERG1 is also located within the cell interior.) The single point brightness was determined within the sarcolemma as described in the Methods section. Only a low amount of immunofluorescence intensity, representing ERG1 protein, was detected in the sarcolemma of sections from young people (Fig. 2E). ERG1 immunofluorescence intensity was more obvious in the sarcolemma of sections from the older 
healthy adult samples (Fig. 2F) and was dramatically greater in the samples from older cachectic adults (Fig. 2G). When single point brightness within the sarcolemma was measured and compared, we found that ERG1 immunofluorescence intensity was 53.3\% $(p<0.05)$ greater in the samples from cachectic people than in the samples from age-matched healthy people (Fig. $2 \mathrm{H})$. When the ERG1 fluorescent intensity was compared in healthy young and healthy aged people, we discovered that there was a greater amount $(10.6 \%)$ of ERG1 signal in the muscle of the healthy aged people than in that of the young; however, this difference was not statistically significant (Fig. $2 \mathrm{H}$ ). Note, the representative muscle section from the cachectic person has small areas of intense staining which do not appear in the samples from healthy persons nor to the same extent in all of the muscle sections from the cachectic people.

The ERG1 Channel Protein is Detected at a Greater Abundance in Cachectic than in Non-Cachectic Muscle. The ERG1 protein is not an abundant protein in heart and is even less abundant in skeletal muscle [22-24, 30,34]; thus, the amount of human tissue necessary for an immunoblot is difficult to obtain, and isolation of the ERG1 protein is further complicated by how labile it is to proteolysis. However, we were able to acquire limb skeletal muscle from humans undergoing above-the-knee leg amputations. Three of these patients had normal BMIs $(\mathrm{BMI}>24)$ while three of the patients were cachectic, presenting with BMls less than 18. We immunoblotted concentrated skeletal muscle membranes from these patients (Fig. 3A) using the upper portion of the PVDF membrane and stained the lower portion of the PVDF membrane with Coomassie Blue to show that equal amounts of protein were loaded into each lane (Fig. 3B). The immunoblot demonstrated that glycosylated forms of the ERG1 protein $[24,30]$ were detectable in all of the muscles, but were $38 \%$ more abundant in the muscle from the cachectic patients $(p=0.09 ; \mathrm{Fig} .3 \mathrm{~A}-\mathrm{C})$. Although it is general practice to set a statistical significance level as $p<0.05$, we feel that a $p$ value equal to 0.09 denotes a real difference in this data set because the sample size is rather small $(n=6)$. Thus, we conclude that the ERG1 protein is more abundant in the cachectic patients. We also immunoblotted Rectus abdominis muscle from a normal patient (Fig. 3D, lane 1) and from two cachectic persons with BMls less than 18 (Fig. 3D, lanes 2 and 3) as well as a sample of esophagus muscle from a person suffering with esophageal cancer (Fig. 3D, lane 4). Obviously, with these latter four samples, we do not have enough sample data for statistical inference. We can only conclude that ERG1 protein was detected in all of these samples, with the appearance of lower ERG1 protein abundance in the one sample from a non-cachectic patient.

ERG1 Protein Localizes Between Bands of Myosin Protein. To further explore ERG1 localization in skeletal muscle cells, we decided to explore how ERG1 fluorescence intensity would be detected in relation to that of myosin (Fig. 4). Because the ERG1 protein is not very prominent in the membrane, it was necessary to use a Tyramide Boost with Alexa Fluor 488 (Invitrogen; Carlsbad, CA) amplification kit to image the ERG1 along with the very prominent myosin fluorescence intensity. Further, to optimize the technique and develop this figure it was necessary that we work with more easily-accessed rat muscle rather than human tissue. As control, longitudinal sections from old rat Gastrocnemius muscle were immuno-stained without primary antibody (Figs. 4A red and 4B green). The absence of green or red fluorescence in these sections demonstrated that any red or green fluorescence in the other matched images is a product of 
primary antibody binding. Muscle sections were also immuno-stained using a mouse antibody (MF20) specific for myosin (Fig. 4C). Then the same muscle sections were co-immunostained with rabbit primary antibody specific for ERG1 (Fig. 4D). This was followed by incubation with an Alexa-fluor 568 red labeled secondary anti-mouse IgG antibody and the labeled goat anti-rabbit secondary antibody supplied with the Tyramide kit. When these images are merged, we see that the fluorescence intensities of the ERG1 and myosin do not co-localize, suggesting a lack of overlap of these proteins in the sample. Indeed, the ERG1 fluorescence is detected in a smaller space between the larger areas (i.e., "stripes") of myosin fluorescence. The pattern is confirmed by a profile plot of a multiple line scan data taken from a representative image of superimposed red and green fluorescence (Fig. 4E) which reveals that the fluorescence emission intensities alternated for ERG1 and myosin proteins. Indeed, the Pearson correlation statistic of the profile data (red compared to green fluorescence) for this single representative image was -0.628 . This pattern suggests that ERG1 is located in connection with the I-band (i.e., the area of thin filaments [actin] that is not superimposed by the thick filaments [myosin]). Both the Z-lines and ttubules are located in the I-band. The t-tubules are located on top of the sarcomere within triads (composed of a t-tubule and two terminal cisternae of the sarcoplasmic reticulum on either side of the ttubule) at each border of the I-band. Therefore, although not definitive evidence, this work does show that ERG1 is located in the l-band and potentially could be located in the t-tubules of skeletal muscle as it is in cardiac muscle $[23,34]$.

Localization of ERG1 Immunofluorescence in Skeletal Muscle is Consistent with a T-tubule Distribution. Because ERG1 protein is known to be located in the t-tubules of cardiac muscle [34], we coimmunostained rat muscle sections for ERG1 and a-actinin, a marker for the Z-line where t-tubules are detected [35]. Thus, rat Gastrocnemius muscle was cryo-sectioned longitudinally and the sections were co-immunostained using a rabbit primary antibody specific for ERG1 protein and a mouse primary antibody specific for a-actinin followed by an Alexa-fluor 568 red labeled secondary anti-mouse IgG antibody and a Tyramide SuperBoost kit with a 488 green labeled secondary anti-rabbit IgG antibody. Red and green fluorescences were absent from sections immunostained without primary antibody (Fig. 5A), demonstrating that detected fluorescence was a result of primary antibody activity. Sections reveal aactinin immunofluorescence (red) and ERG1 immunofluorescence (green) in patterns expected for each (Fig. 5B,C). Merged fluorescent confocal images of co-immunostained muscles revealed that the a-actinin (red) fluorescence was located within ERG1 (green) fluorescence, but that these proteins did not colocalize because yellow fluorescence is essentially absent in the micrograph (Fig. 5D). This interpretation is strengthened by fluorescence intensity profile measurements (Fig. $5 \mathrm{E}$ ) which demonstrated that the ERG1 (green) and DHPR (red) signals followed the same pattern through the demarcated region (Fig. 5D, white line) with the red signal falling within the green. The Pearson correlation statistic of the profile data (red compared to green fluorescence) for this single sample representative image was 0.8667 , demonstrating that the two fluorescent signals followed a similar pattern with the green signal being broader than the red. The pattern is consistent with the interpretation that ERG1 protein is located in ttubules which lie above the plane of the sarcomere on either side of the Z-line. Indeed, t-tubules are reported to be $\sim 40-100 \mathrm{~nm}$ in diameter (specifically $85.4 \pm 4.9 \mathrm{~nm}$ for rat muscle [36]) which falls below 
the optical spatial resolution limit $(\sim 200 \mathrm{~nm})$ of our equipment. The lack of appropriate optical resolution did not allow us to resolve completely the green and red fluorescence emission intensities which appear to overlap in the micrograph.

To explore further the localization of the ERG1 protein in skeletal muscle, we co-immunostained longitudinal skeletal muscle sections for ERG1 and DHPR, a marker for t-tubules [37]. Thus, old rat Gastrocnemius muscle was cryo-sectioned longitudinally and the sections were co-immunostained using a rabbit primary antibody specific for ERG1 protein and a mouse primary antibody specific for the t-tubule marker protein DHPR followed by an Alexa-fluor 568 red labeled secondary anti-mouse IgG antibody and an Alexa-fluor 488 green labeled secondary anti-rabbit lgG antibody. Red and green fluorescences were absent from sections immunostained without primary antibody (Fig. 6A), demonstrating that detected fluorescence is a result of primary antibody activity. Sections revealed DHPR immunofluorescence (red) in a pattern consistent with t-tubule membranes as expected (Fig. 6B). The green fluorescence in immunostained sections revealed that ERG1 immunofluorescence was consistent with a pattern of ttubule distribution (Fig. 6C, these results were consistent with human RA muscles, data not shown). Merged fluorescent confocal images of co-immunostained muscles showed that ERG1 (green) and DHPR (red) immunofluorescences co-localized (yellow) suggesting that ERG1 localization is consistent with ttubule distribution (Fig. 6D). This interpretation is strengthened by fluorescence intensity profile measurements (Fig. 6E) which demonstrated that the ERG1 (green) and red (DHPR) signals followed the same pattern through the demarcated region (Fig. 6D, white line) of the t-tubules. The Pearson correlation statistic of the profile data (red compared to green fluorescences) for this single sample representative image was 0.928 , demonstrating that the two fluorescent signals were similarly localized.

\section{Discussion}

Detection of the ERG1 splice variant proteins, $A$ and $B$, has been reported in the heart of numerous species where they have been shown to form hetero-multimeric potassium channels which contribute to late phase repolarization of the action potential [17-19, 22,23]. ERG1A has also been detected in the skeletal muscle of rats and mice with the highest abundance being in atrophic relative to normal muscle [24]. To our knowledge, this is the first report of the ERG1 protein in human skeletal muscle. Here we demonstrate that the human ERG1 protein is detected in the sarcolemmal membrane of human Rectus abdominis muscle, showing a localization pattern similar to that of the sarcolemmal membrane marker dystrophin. With this information, we explored the relative abundances of ERG1 in the sarcolemmal membranes of Rectus abdominis muscle samples from young healthy, older healthy, and older cachectic humans. Using images from tissues sections treated with immuno-histochemical methods, we report that ERG1 fluorescence (using single point brightness data [31, 32]) is statistically more abundant in the sarcolemma of the Rectus abdominis muscle of cachectic humans than in the same muscle of healthy age-matched people. Interestingly, the representative muscle section from the aged, cachectic person (Fig. 2D) has small areas of intense staining with shape and placement consistent with myonuclei. These areas of intense staining are less obvious in muscle from healthy persons where ERG1 fluorescence is less intense. Indeed, it is possible that HERG1 protein is located in the myonuclei; however, because of 
their close proximity to the sarcolemma where HERG1 is abundant, it is not possible to draw a definitive conclusion from the current images. This will require more study.

To further explore the abundance of ERG1 protein in cachectic versus normal skeletal muscle, we performed an immunoblot with human tissue. This experiment was complicated by the fact that ERG1 protein is not a prominent protein (even in cardiac tissue) and was further impacted by the fact that ERG1 is very labile to proteolysis [30,38-40]. Thus, it is difficult to get the necessary amount of human skeletal muscle to perform these studies, especially from young healthy people. Initially, we were unable to get tissue from a consistent muscle or age group; however, we discovered that we could purchase skeletal muscle samples taken from people undergoing an above-the-knee amputation and that some of these were cachectic (although only one of the three cachectic patients was reported to have a malignancy; Table 2, see Materials and Methods). We homogenized the muscle samples and then concentrated the membrane fraction by adding a low volume of solubilization buffer. Indeed, we found that ERG1 protein is more abundant in the muscle of the cachectic patients which concurs with our earlier immunoblot data showing that the mouse ERG1a protein is significantly more abundant in the skeletal muscle of cachectic nude mice expressing malignant tumors than in the muscle of healthy control animals [24]. Here, however, the $\mathrm{p}$ value for the Student's t-test, which compares the cachectic with the healthy persons, is below the $p<0.05$ level of significance set earlier. Nonetheless, we conclude that the increased ERG1 protein abundance in the cachectic human samples is real mainly because we have such a small sample size $(n=6)$. Indeed, we conducted a power analysis with our current data to project what sample size would be necessary to detect a significant difference between the tissues from the aged healthy and aged cachectic adults in terms of ERG1 protein abundance and found that an increase in sample size from a total of $n=6$ to $n=12$ would allow us to detect a difference at significance level $p=0.05$ between the two groups with $90 \%$ power. Here, we also decided to immunoblot the remaining human samples we had managed to obtain (Fig. 3D,E); however, the samples are not from consistent muscles, age groups, or disease states. Because this immunoblot includes only one normal Rectus abdominis sample and two that are from cachectic cancer patients, and a fourth sample that is actually from malignant esophageal muscle, we can only report that ERG1A is detected in those tissues. In earlier studies, we also showed that mouse ERG1a protein was more abundant in the atrophic skeletal muscle of unweighted mice and demonstrated that the ERG1a $\mathrm{K}^{+}$channel increased in response to these atrophic insults and contributed to the proteolytic component of the skeletal muscle atrophy that followed [24]. Specifically, we overexpressed plasmid encoding mouse Erg1a in mouse Gastrocnemius muscles and demonstrated that (relative to controls) expression of the muscle-specific atrophy-related E3 ligase, MuRF1, and overall UPP activity increased in response and that muscle fiber cross sectional area significantly decreased in the Merg1a-expressing myofibers. We also inhibited atrophy in unweighted mice by: 1) expressing a dominant negative Merg1a plasmid [25] in their Gastrocnemius muscle; and separately 2) treating the mice with the pharmacologic ERG1 blocker, astemizole (Sigma; St. Louis, MO). Because we find that ERG1 protein is more abundant in cachectic human skeletal muscle (Fig. 2, 3A-C), it is logical to suggest that ERG1 may also contribute to skeletal muscle atrophy in cachectic humans. 
With our IHC single point data, we also demonstrate that ERG1 protein abundance is $10.6 \%$ greater in the Rectus abdominis muscle of healthy older humans than in that of healthy young adults (Fig, 2). Although the difference is not statistically significant, it does suggest that increasing abundance of ERG1 could be involved in the rather slow, natural muscle loss that occurs with age (i.e., sarcopenia [33]). One potential confounder is that our current analyses of immunostained muscle cross sections allow us to evaluate fluorescence only from the sarcolemmal membrane because the immunofluorescence from $t$-tubules is not specifically identifiable in cross sections. It is possible that, if the analysis included the ERG1 protein in the t-tubules, the difference in ERG1 protein abundance detected in the tissues from the young people and those of the healthy older people could become more (or less) pronounced. Indeed, ERG1 protein was found to be more prominent in the t-tubules than in the sarcolemma of cardiac tissue [34]. Thus, we again conducted a power analysis with our current data from one observer (there was no difference found between the observers' data sets; Fig. 2E) to project what sample size would be necessary to detect a significant difference between the tissues from the young and aged healthy adults in terms of ERG1 fluorescence intensity and found that an increase in sample size from a total of 11 (4 young and 7 healthy aged) to 46 would allow us to detect a difference at significance level $p=0.05$ between the two groups with $90 \%$ power. Obviously, getting Rectus abdominis samples from 46 (especially 23 young, healthy) humans would be difficult; however, because the number is not completely unreasonable, it suggests that the difference in ERG1 protein abundance between the two groups may indeed be more important than the small sample size allows us to conclude. More interestingly, it leads us to suspect that the ERG1 protein would be even more abundant in the skeletal muscle of sarcopenic (aged and frail) people than in that of the healthy aged individuals. Indeed, we showed that ERG1A protein is more abundant in the Gastrocnemius muscles of aged (30 months) than in young (3 months) rats [41]. Another matter of note is that the ERG1 antibody we used does not allow us to distinguish between Erg1a and 1b alternative splice variants; however, we have not detected ERG1b protein or mRNA in mouse skeletal muscle [24] and do not expect it to be detected in human skeletal muscle. Further, ERG1b is often considered to be "cardiac specific" [18, 19]; although there are reports of it being found in brain [20] and some cancer cells [21].

We also present data here that demonstrate that ERG1 protein is located in the sarcolemmal membranes of human RA muscle (Fig. 1). The mottled appearance of fluorescence in the cell interior suggests that ERG1 protein is potentially located in the sarcoplasm of human skeletal muscle. Indeed, it is detected in areas consistent with I band localization (the area between myosin thick filaments, Fig. 4, and on either side of a-actinin, Fig. 6) and is shown to co-localize with the known t-tubule marker, DHPR (Fig. 5). Although, to our knowledge, this is the first demonstration of ERG1 detection specifically in the sarcolemma or within the I-band area of human skeletal muscle sarcomeres, the localization is not surprising because the ERG1 protein has been detected in conjunction with these structures in cardiac tissues $[23,34]$. Specifically, using immunohistochemistry and confocal microscopy, Rasmussen and colleagues (2004; [34]) reported detection of prominent ERG1 protein in the t-tubules of rat atria and ventricles, which correlated well with their detection of the known cardiac t-tubule marker protein DHPR a2-subunit; however, they did not report that the ERG1 and DHPR a2-subunit proteins co-localize within 
the structure. As expected, they also detected the DHPR a2-subunit protein in the peripheral sarcolemma along with the ERG1 protein, reporting relatively less prominent levels of the ERG1 protein in the sarcolemma (concentrated near invaginations to t-tubules) than in the t-tubules. TEM confirmed these findings, revealing lower abundances of ERG1 protein in discretely localized clusters within the sarcolemmal membrane. Indeed, the presence of ERG1 clusters in the sarcolemma may explain the inconsistent localization pattern of ERG1 we find within the sarcolemma (Fig. 1).

The laboratory of Dr. Gail Robertson (Univ. of WI, Madison) has studied this channel protein extensively and, using biotinylation studies with an HEK-293 cell line and immunoprecipitation studies in both canine and human heart, has elegantly demonstrated that mature glycosylated forms of both ERG1a and 1b proteins are expressed on the cell surface and that ERG1a and 1b proteins co-localize to the same subcellular compartment in canine myocytes, having a punctate "Z-line-like" pattern characteristic of ttubular localization. Three dimensional images constructed using two dimensional images of canine ventricle stained concurrently for ERG1a and myosin binding protein C (MyBP-C) revealed that ERG1 is localized in I bands (which contain both Z-lines and T-tubules); further, the ERG1 signal was shown to extend in lines from the cytoplasmic membrane to the interior, consistent with a t-tubular distribution [23]. The Robertson lab has further demonstrated that human ERG1a and $1 \mathrm{~b}$ proteins comprise the cardiac ERG1 channel and that these proteins assemble co-translationally through interactions of their $\mathrm{N}$-termini [22].

The obvious question is: What does ERG1 do in the atrophying skeletal muscle? Our earlier data suggest strongly that it modulates proteolytic activity in mouse skeletal muscle. Specifically, we showed that over expression of Merg1a in mouse skeletal muscle increases expression of the MuRF1 E3 ligase component of the UPP and enhances UPP activity [24], but interestingly it does not affect the expression of the Mafbx/ATROGIN1 E3 ligase [26, 27] which is considered to be an important marker of atrophy in skeletal muscle [42]. We do not yet know the full signaling mechanism for the enhanced Murf1 expression or the increased MuRF1 protein levels that follow Merg1a expression [24, 31, 32]. Of course, in these studies the Merg1a gene was over-expressed and it can be argued that these results may not reflect true physiological effects. However, when the channel was blocked in unweighted mice, by both expression of a dominant negative subunit and treatment with the known pharmacological ERG1 blocker, astemizole, the atrophy (decrease in cross sectional area) was inhibited [24]. These data support the idea that ERG1 may contribute to atrophy.

A more evident question is: Does ERG1 affect excitation contraction coupling? ERG1 is a membrane bound voltage-gated ion channel shown to be located on t-tubules in heart [23, 24]. Our data suggest it may be located on t-tubules in skeletal muscle. Thus, it is possible that ERG1 could affect transduction of signal from the sarcolemmal membrane to the contraction mechanism; that is, it may have a role in excitation contraction coupling and its increased abundance might modulate this event. Indeed, we have recently shown that increased ERG1A expression in $\mathrm{C}_{2} \mathrm{C}_{12}$ myotubes results in an increased intracellular calcium concentration [43]. However, the action potential duration in skeletal muscle is much shorter than in cardiac muscle (2-5 ms vs. $200-400 \mathrm{~ms}$ ) and ERG1 has a very slow rate of activation which is not 
likely to be relevant to action potential duration in skeletal muscle. So it is not likely that ERG1 affects the skeletal muscle action potential. Further, the relatively positive activation threshold for ERG1 ( -30 mV; [44]) precludes its regulation of resting membrane potential $(\sim-80 \mathrm{mV}$; [45]) in skeletal muscle. However, ERG1 could potentially regulate DHPR activity by uncoupling it from excitation contraction coupling as is known to occur with aging [33]. The location of the ERG1 protein in the t-tubules certainly places it in the proximity of the calcium release mechanism [23,34], suggesting that ERG1 may interact with/modulate this complex. Indeed, uncoupling of DHPR and RYR proteins [46], progressive disorganization and spatial rearrangement of the excitation contraction coupling apparatus [47], and abnormalities in calcium release [48] are shown to occur in skeletal muscle with aging [33] and disease [2]. Further studies are needed to determine whether an increase in ERG1 channel expression contributes or responds to these effects.

\section{Conclusions}

In summary, here we report detecting significantly more ERG1 immunofluorescence and a greater ERG1 protein abundance in the sarcolemma of skeletal muscle sections from cachectic people than in muscle sections from age matched healthy humans. Further, we reveal that ERG1 was detected more prominently in the skeletal muscle of aged than young humans. This is consistent with our findings with which we show that ERG1 protein was more abundant in the atrophying than normal skeletal muscle of mice [24] and also more abundant in the skeletal muscle of aged versus young rats [41]. We have shown that ERG1a modulates myofiber size in atrophying mouse muscle [24] and expect that it functions similarly in human muscle, but this needs to be explored. We also demonstrate that, as in cardiac tissue, ERG1 protein localizes to the sarcolemma and supply evidence which suggests it also exists in the t-tubule membranes where it has the added potential to affect excitation-contraction coupling and calcium release through modulation of DHPR and ultimately RYR and/or IP3 receptors. Obviously, this localization needs to be further explored and confirmed with TEM analyses. Because skeletal muscle atrophy is a prominent health concern for injured, ill and elderly people, it is important that research on the skeletal muscle ERG1 $\mathrm{K}^{+}$channel continue.

\section{Abbreviations}

ANOVA - analysis of variance

BSA - bovine serum albumin

DHPR - dihydropyridine receptor

DN-Merg1a - dominant negative murine ether-a-go-go related gene

DSHB - Developmental Studies Hybridoma Bank

ERG1 - ether-a-go-go related gene 1 
FBN - F1 hybrid of Fischer 344 (F344) and Brown Norway rats

GS - Gastrocnemius (muscle)

IHC - immunohistochemistry

Merg (MERG) - mouse ether-a-go-go related gene

PBS - phosphate buffered saline

$R A-$ Rectus abdominis (muscle)

$R T$ - room temperature

SEM - standard error of the mean

UPP - ubiquitin proteasome pathway

WT - wild type

\section{Declarations}

Ethics Approvals and Consents. Human Skeletal Muscle. For figures 1 and 2 (Table 1), healthy subjects were control donors who were undergoing elective laparotomy for non-neoplastic and non-inflammatory diseases. Cachectic patients affected with colorectal, esophago-gastric or pancreatic cancers were enrolled for a project approved by the Ethical Committee for Clinical Experimentation of Padova (protocol number 3674/AO/15). All enrolled subjects were volunteers who signed written informed consent forms. For figure 3, the skeletal muscle samples from limbs amputated just above the knees were purchased from The Collaborative Human Tissue Network (CHTN), Midwestern Division (Ohio State University). The human Rectus abdominis muscle $(R A)$ samples and the malignant esophageal muscle sample used for the immunoblot (Figure 3 D, E; Table 2) were purchased from the SIU School of Medicine Tissue Bank, a Simmons Cancer Institute funded program. All purchased samples were de-identified before approved use and approval to use was waived by the SIU IRB. Rat Skeletal Muscle. The FBN rat (National Institute of Aging Rodent Service) muscles used for the immunohistochemistry were generous frozen gifts from Dr. Don Caspary (Southern Illinois University School of Medicine; Springfield, IL). Dr. Caspary's animal studies are all approved by the SIU School of Medicine Institutional Animal Care and Use Committee.

Consent for Publication. Not applicable.

Availability of Data and Materials. The datasets used and/or analysed during the current study are available from the corresponding author on reasonable request.

Competing Interests. The authors declare that they have no competing interests. 
Funding. This work was supported in part by Southern Illinois University School of Medicine (Start Up funding to ALP) and by a REACH Award from the SIU Carbondale Office of Sponsored Research received by LBA. The work was also supported in part by the Italian Society for Cancer Research (AIRC grant number 17388 to M Sandri) and by Austrian national co-financing of the Austrian Federal Ministry of Science and Research; Ludwig Boltzmann Society (Vienna, Austria) to H Kern. PK would like to acknowledge National Science Foundation (CHE 0748676), NIH (GM 106364) for partial financial support of this research. The authors wish to thank Dr. Don Caspary who graciously provided FBN rat muscles. Human Rectus abdominis muscle samples and the malignant esophageal muscle sample were purchased from the SIU School of Medicine Tissue Bank, a Simmons Cancer Institute funded program. Other investigators may have received samples from these same tissue specimens. The skeletal muscle samples from limbs amputated just above the knees of patients with either a normal or low (<18) BMI were purchased from The Collaborative Human Tissue Network (CHTN), Midwestern Division. UC thanks the IRCCS Fondazione Ospedale San Camillo, Venice, Italy and the A\&C M-C Foundation for Translational Research for scientific support.

Author's Contributions. SZ contributed to development of the project, to structuring of all figures, to writing of the initial manuscript, and helped with all subsequent revisions; she also managed human samples and contributed to H\&E stains for Figures 1 and 2. MS contributed to acquisition of the human samples used in Figures 1 and 2; he also contributed to the initial writing of the manuscript and all subsequent revisions. JLC guided the immunostaining necessary for all figures and performed the confocal microscopy which captured the images displayed in Figure 6; he also aided in writing revisions of the manuscript. RPB performed the confocal microscopy necessary to capture the images in Figures 4 and 5; he also aided in revision of the final manuscript. LBA contributed to cryo-sectioning, immunostaining, and fluorescent microscopy of all muscle sections; he also performed the single point analysis (observer 1) shown in Figure 2 and contributed to review and revision of the manuscript. BAC contributed to sectioning and immunostaining of all muscle tissue. CDL performed the single point analysis (observer 2) shown in Figure 2; she also contributed strongly to development of the experimental design and performed all statistical analyses as well as reviewed and revised the manuscript. GHH participated in development of the project and contributed to the initial writing of the manuscript as well as subsequent revisions. HK contributed to development of the original project and aided with revision of the manuscript. RS contributed to acquisition of the human samples used in Figures 1 and 2; she also contributed to revision of the manuscript. BR prepared and sectioned human muscle tissue and contributed to performance of the H\&E stains. SM and GD harvested the human muscle tissues used in Figures 1 and 2 and also contributed to revision of the manuscript. JKD contributed to development of the project, to writing of the initial manuscript, and helped with all subsequent revisions. PK acquired the confocal microscope used to capture the images in Figures 4 and 5; he mentored the performance of the confocal microscopy necessary to capture these images, and also aided in revision of the final manuscript. UC mentored development of the project, contributed to the writing of the initial manuscript, and helped with all subsequent revisions. ALP developed the project, performed or guided the immunostaining displayed in all figures, guided the microscopy performed in Figures 1 and 2, performed 
the immunoblots and consequent analysis displayed in Figure 3, wrote the initial manuscript, and crafted the final submitted draft. All authors read and approved the final manuscript.

Acknowledgements. Not applicable.

\section{References}

1) Taylor MJ1,2,3, Schils S4, Ruys AJ1. Home FES: An Exploratory Review. Eur J Transl Myol. 2019;29(4):8285. DOI:10.4081/ejtm.2019.8285. eCollection 2019 Oct 29.

2) Wing SS, Lecker SH, Jagoe RT. Proteolysis in illness-associated skeletal muscle atrophy: from pathways to networks. Critic Rev Clin Lab Sci. 2011;48:49-70. DOI:10.3109/10408363.2011.586171.

3) Ryall JG, Schertzer JD, Lynch GS. Cellular and molecular mechanisms underlying age-related skeletal muscle wasting and weakness. Biogerontology. 2008;9:213-228. DOI:10.1007/s10522-008-9131-0.

4) Janssen I., Heymsfield SB, Ross R. Low relative skeletal muscle mass (sarcopenia) in older persons is associated with functional impairment and physical disability. J Am Geriatrics Soc. 2002;50:889-896. DOI:10.1046/j.1532-5415.2002.50216.x.

5) Marzetti E, Calvani R, Bernabei R, Leeuwenburgh C. Apoptosis in skeletal myocytes: A potential target for interventions against sarcopenia and physical frailty - a mini-review. Gerontology. 2012;58:99-106. DOI:10.1159/000330064.

6) Puthucheary $Z$, Harridge $S$, Hart N. Skeletal muscle dysfunction in critical care: wasting, weakness, and rehabilitation strategies. Crit Care Med. 2010;38:S676-82. DOI:10.1097/CCM.0b013e3181f2458d.

7) Bossola M, Pacelli F, Tortorelli A, Rosa F, Battista Doglietto G. Skeletal muscle in cancer cachexia: the ideal target of drug therapy. Current Cancer Drug Targets. 2008;8:285-98.

DOI:10.2174/156800908784533463.

8) Damrauer JS, Stadler ME, Acharyya S, Baldwin AS, Couch ME, Guttridge DC. Chemotherapy-induced muscle wasting: association with NF-kB and cancer cachexia. Eur J Transl Myol. 2018;28(2):7590. DOI:10.4081/ejtm.2018.7590. eCollection 2018 Apr 24.

9) Coletti D. Chemotherapy-induced muscle wasting: an update. Eur J Transl Myol. 2018;28(2):7587. DOI:10.4081/ejtm.2018.7587. eCollection 2018 Apr 24.

10) Zinna EM, Yarasheski KE. Exercise treatment to counteract protein wasting of chronic diseases. Curr Opin Clin Nutr Metabol Care. 2003;6:87-93. DOI:10.1097/01.mco. 0000049042.06038.b7

11) Patel DI, Abuchowski K, Sheikh B, Rivas P, Musi N, Kumar AP. Exercise preserves muscle mass and force in a prostate cancer mouse model. Eur J Transl Myol. 2019;29(4):8520.

DOI:10.4081/ejtm.2019.8520. eCollection 2019 Oct 29. 
12) Djemai H, Hassani M, Daou N, Li Z, Sotiropoulos A, Noirez P, Coletti D. Srf KO and wild-type mice similarly adapt to endurance exercise. Eur J Transl Myol. 2019;29(2):8205. DOI:10.4081/ejtm.2019.8205. eCollection 2019 May 7.

13) Fareed MU, Evenson AR, Wei W, Menconi M, Poylin V, Petkova V, Pignol B, Hasselgren PO. Treatment of rats with calpain inhibitors prevents sepsis-induced muscle proteolysis independent of atrogin1/MAFbx and MuRF1 expression. Am J Physiol Regul Integr Comp Physiol. 2006;290:R1589-97. DOI:10.1152/ajpregu.00668.2005.

14) Baoge L, Van Den Steen E, Rimbaut S, Philips N, Witvrouw E, Almqvist KF, Vanderstraeten G, Vanden Bossche LC. Treatment of skeletal muscle injury: A review. ISRN Orthopedics. 2012. DOI:10.5402/2012/689012. eCollection 2012.

15) Guasconi V, Puri PL. Epigenetic drugs in the treatment of skeletal muscle atrophy. Curr Opin Clin Nutr Metab Care. 2008;11:233-41. DOI:10.1097/MCO.0b013e3282fa1810.

16) Smith RC, Lin BK. Myostatin inhibitors as therapies for muscle wasting associated with cancer and other disorders. Curr Opin. 2013;7(4):352-60. DOI:10.1097/SPC.0000000000000013.

17) Curran ME, Splawski I, Timothy KW, Vincent GM, Green ED, Keating MT. A molecular basis for cardiac arrhythmia: herg mutations cause long QT syndrome. Cell. 1995;80:795-803. PMID:7889573.

18) London B, Trudeau MC, Newton KP, Beyer AK, Copeland NG, Gilbert DJ, Jenkins NA, Satler CA, Robertson GA. Two isoforms of the mouse Ether-a-go-go-related gene coassemble to form channels with properties similar to the rapidly activating component of the cardiac delayed rectifier $\mathrm{K}^{+}$current. Circ Res. 1997;81:870-878. DOI:10.1161/01.RES.81.5.870.

19) Lees-Miller JP, Kondo C, Wang L, Duff HJ. Electrophysiological characterization of an alternatively processes erg K+ channel in mouse and human hearts. Circ Res. 1997;81:719-726.

DOI:10.1161/01.RES.81.5.719.

20) Guasti L, Cilia E, Crociani O, Hofmann G, Polvani S, Becchetti A, Wanke E, Tempia F, Arcangeli A. Expression pattern of the ether-a-go-go-related (ERG) family proteins in the adult mouse central nervous system: Evidence for coassembly of different subunits. J Comp Neurol. 2005;491(2):157-174. DOI:10.1002/cne.20721.

21) Babcock JJ, Li M. hERG channel function: beyond long QT. Acta Pharm Sinica. 2013;34:329-335. DOI:10.1038/aps.2013.6.

22) Phartiyal $P$, Jones EM, Robertson $G$. Heteromeric assembly of human ether-a-gogo-related (hERG) $K^{+}$ channels occurs cotranslationally via N-terminal interactions. J Biol Chem. 2007; 282:9874-9882. DOI:10.1074/jbc.M610875200. 
23) Jones EMC, Roti EC, Wang J, Delfosse $S A$, Robertson GA. Cardiac $\mathrm{I}_{\mathrm{Kr}}$ channels minimally comprise hERG1a and 1b subunits. JBC. 2004;279(43):44690-44694. DOI:10.1074/jbc.M408344200.

24) Wang $X$, Hockerman GH, Green $3^{\text {rd }} \mathrm{HW}$, Babbs $\mathrm{CF}$, Mohammad SI, Gerrard D, Latour MA, Hannon KM, Pond AL. Merg1a $\mathrm{K}^{+}$channel induces skeletal muscle atrophy by activating the ubiquitin proteasome pathway. FASEB J. 2006;20(9):1531-3. DOI:10.1096/fj.05-5350fje.

25) Selyanko AA, Delmas P, Hadley JK, Tatulian L, Wood IC, Mistry M, London B, Brown DA. Dominantnegative subunits reveal potassium channel families that contribute to M-like potassium currents. $J$ Neurosci. 2002;22: RC212-217.

26) Hockerman GH, Dethrow NM, Hameed S, Doran M, Jaeger C, Wang W-H, Pond AL. The Ubr2 gene is expressed in skeletal muscle atrophying as a result of hind limb suspension, but not Merg1a expression alone. Eur J Trans Myol. 2014; 24(3):173-179. DOI:10.4081/ejtm.2014.3319.

27) Pond AL, Nedele C, Wang W-H, Wang X, Walther C, Jaeger C, Bradley KS, Du H, Fujita N, Hockerman $\mathrm{GH}$, Hannon KM. The MERG1a channel modulates skeletal muscle Murf1, but not MAFbX, expression. Muscle \& Nerve. 2013;49(3):378-388. DOI:10.1002/mus.23924.

28) Fearon K, Trasser F, Anker SD, Bosaeus I, Bruera E, Fainsinger RL, Jatoi A, Loprinzi C, MacDonald N, Mantovani G, David M, Muscaritoli M, Ottery F, Radbruch L, Ravasco P, Walsh D, Wilcock A, Kaasa S, Baracos VE. Definition and classification of cancer cachexia: an international consensus. Lancet Oncol. 2011;2:489-495.

29) Fischman DA. Immunochemical analysis of myosin heavy chain during avian myogenesis in vivo and in vitro. J Cell Biol. 1982;95(3):763-70.

30) Pond AL, Petrecca K, Van Wagoner DR, Shrier A, Nerbonne JM. Expression of distinct ERG1 proteins in rat, mouse and human heart: Relation to functional $\mathrm{I}_{\mathrm{Kr}}$ channels. JBC. 2000;275:5997-6006. DOI:10.1074/jbc.275.8.5997.

31) Schneider CA, Rasband WS, Eliceiri KW. "NIH Image to ImageJ: 25 years of image analysis", Nature Methods. 2012;9(7):671-675. PMID 22930834.

32) Cheatwood JL, Emerick AJ, Schwab ME, Kartje GL. Nogo-A expression after focal ischemic stroke in the adult rat. Stroke. 2008; 39:2091-2098. DOI:10.1161/STROKEAHA.107.507426.

33) Miljkovic N, Lim J-Y, Miljkovic I, Frontera WR. Aging of skeletal muscle fibers. Ann Rehabil Med. 2015;39(2):155-162. DOI:10.5535/arm.2015.39.2.155.

34) Rasmussen HB, Moller M, Knaus H-G, Jensen BS, Olesen S-P, Jorgensen NK. Subcellular localization of the delayed rectifier K+ channels KCNQ1 and ERG1 in the rat heart. Am J Physiol Heart Circ Physiol. 2004;286:H1300-H1309. DOI:10.1152/ajpheart.00344.2003. 
35) Protasi F. Structural interaction between RYRs and DHPRs in calcium release units of cardiac and skeletal muscle cells. Front Biosci. 2002;7:d650-658. PMID:11861217.

36) Jayasinghe ID, Launikonis BS. Three-dimensional reconstruction and analysis of the tubular system of vertebrate skeletal muscle. J Cell Science 2013;126:4048-4058.

37) Goll DE, Dayton WR, Singh I, Robson RM. Studies of the a-actinin/atin interactions in the Z-disk by using calpain. J Biol Chem 1991;266(13):8501-8510.

38) Wang X, Xu R, Abernathy GT, Taylor J, Alzghoul MB, Hannon K, Hockerman GH, Pond AL. Kv11.1 K channel composition varies developmentally in mouse heart. Dev Dynamics. 2007;237:2430-2437. DOI:10.1002/dvdy.21671.

39) Van Wagoner DR, Pond AL, Lamorgese M, Rossie SS, McCarthy PM, Nerbonne JM. Atrial L-type Ca2 ${ }^{+}$ currents and human atrial fibrillation. Circ Res. 1999;85:428-436. DOI:10.1161/01.RES.85.5.428

40) Wang Z, Feng J, Shi J, Pond AL, Nerbonne JM, Nattel S. Potential molecular basis of different physiological properties of transient outward current in rabbit and human atrial myocytes. Circ Res. 1999;84:551-561. DOI:10.1161/01.RES.84.5.551

41) Anderson LB, Latour CD, Khader O, Massey BH, Cobb B, Pond AL. Ether-a-go-go related gene-1a potassium channel abundance varies within specific skeletal muscle fiber type. Euro J Transl Myol 2019;29(3):8402. DOI:10.4081/ejtm.2019.8402. eCollection 2019 Aug 2.

42) Bonaldo P, Sandri M. Cellular and molecular mechanisms of muscle atrophy. Disease Models \& Mechs. 2013;6:25-39. DOI:10.1242/dmm.010389.

43) Whitmore C, Pratt EPS, Anderson LB, Bradley K, Latour SM, Hashmi MN, Urazaev AK, Weilbaecher R, Davie JK, Wang W-H, Hockerman GH, Pond AL. The ERG1 a potassium channel increses basal intracellular concentration and calpain activity in skeletal muscle cells. Skeletal Muscle. 2020;10(1). DOI: 10.1186/s13395-019-0220-3.

44) Sanguinetti MC, Curran ME, Spector PS, Keating MT. Spectrum of HERG $K^{+}$-channel dysfunction in an inherited cardiac arrhythmia. PNAS USA. 1996;93:2208-2212. PubMed ID8700910

45) Pederson TH, Riisager A, de Paoli FV, Cehn T-Y, Nielsen OB. Role of physiological ClC-1Cl- ion channel regulation for the excitability and function of working skeletal muscle. J Gen Physiol. 2016;147(4):291308. DOI:10.1085/jgp.201611582.

46) Renganathan $M$, Messi $M L$, Delbono O. Dihydropyridine receptor-ryanodine receptor uncoupling in aged skeletal muscle. J Membr Biol. 1997;157:247-253. DOI:10.1007/s002329900233.

47) Boncompagni S, d'Amelio L, Fulle S, Fanò G, Protasi F. Progressive disorganization of the excitationcontraction coupling apparatus in aging human skeletal muscle as revealed by electron microscopy: a 
possible role in the decline of muscle performance. J Gerontol A Biol Sci Med Sci. 2006;61(10):995-1008. DOI:10.1093/gerona/61.10.995.

48) Delbono 0. Regulation of excitation contraction coupling by insulin-like growth factor- 1 in aging skeletal muscle. J Nutr Health Aging. 2000;4:162-164. PMID:10936903.

\section{Tables}

Table 1. Human subjects providing samples for immunohistochemistry.

$\begin{array}{lllll}\text { Sample Source } & \text { Tumor site } & \text { Sex } & \text { Age (yrs) } & \text { BMI }\end{array}$

Cachectic patients; $\mathrm{n}=5$; Mean Age $\pm \mathrm{SD}=68.2 \pm 11.4 \mathrm{yrs}$

1

$\begin{array}{llll}\text { Esophagus } & \mathrm{M} & 65 & 23.7\end{array}$

2

Colon $\quad \mathrm{M} \quad 88$

25

3

Pancreas $\quad$ F $\quad 66$

21.5

4

Pancreas

F

59

24.7

5

Pancreas

F

63

20

Healthy aged; $n=7$; Mean Age \pm SD $=67.9 \pm 9.1$ yrs

M

63

30

7

M $\quad 62$

8

$\begin{array}{ll}M & 68\end{array}$

24.6

9

M

61

22.7

10

F

62

nd

11

F $\quad 86$

24.6

12

F

73

29.6

Healthy young; $\mathrm{n}=4$; Mean Age $\pm \mathrm{SD}=25.8 \pm 2.2 \mathrm{yrs}$

13

14

15

16
M 24

F 29

F 25

$\mathrm{F}$

25

Note. Abbreviations: yrs = years; $\mathrm{n}=$ number of subjects.

Table 2. Human subjects providing samples for immunoblots. 
Limb Tissue from patients with BMI $>25$; $=6$; Mean Age \pm SD $=60 \pm 3.6$ yrs

None

F

58

2

None

$\mathrm{F}$

67

3

None

M

55

Limb Tissue from Patients with $\mathrm{BMI}<18$; $\mathrm{n}=3$; Mean Age $\pm \mathrm{SD}=63.7 \pm 0.3$

4

Arm

$\mathrm{F}$

63

5

Esophagus

M

64

6

None

M

64

Rectis abdominis muscle; $\mathrm{n}=3$; Mean Age $\pm \mathrm{SD}=51.3 \pm 7.1$

1

None; $\mathrm{BMI}>25$

$\mathrm{F}$

48

2

tumor site not reported; $\mathrm{BMI}<18$

M

41

3

tumor site not reported; $\mathrm{BMI}<18$

F

65

Esophageal Muscle; $\mathrm{n}=1$

Esophagus $\quad$ M $\quad 59$

Note. Abbreviations: $\mathrm{yrs}=$ years; $\mathrm{n}=$ number of subjects.

\section{Figures}



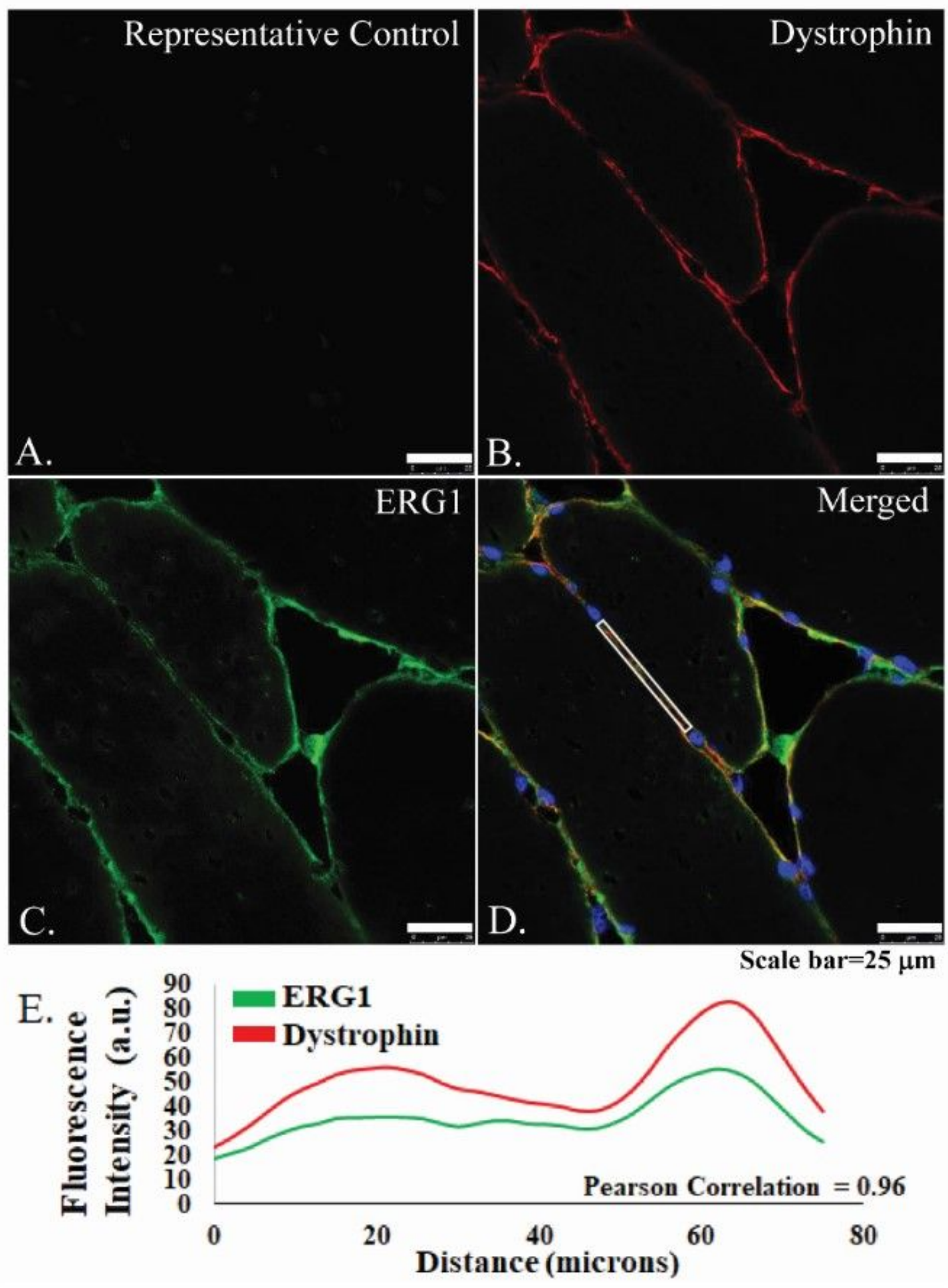

\section{Figure 1}

ERG1 fluorescence localizes to sarcolemma of human Rectus abdominis myofibers as shown by fluorescent immunohistochemistry. A. Fluorescence was absent from sections immuno-stained without primary antibody. B. Fluorescence (red) of dystrophin, a skeletal muscle sarcolemmal membrane marker, was detected in sarcolemmal membrane as expected. C. ERG1 fluorescence (green) was detected in sarcolemmal membrane. D. ERG1 (green) and dystrophin (red) immunofluorescence were both detected 
in the sarcolemmal membrane of human Rectus abdominis muscle. DAPI staining (blue) revealed nuclei. Scale bar is $25 \mathrm{um}$. The white rectangular region is the area in which fluorescence intensity was measured. E. ERG1 (green) and dystrophin (red) fluorescences both localize to the sarcolemmal membrane as evidenced by fluorescence intensities, which follow the same pattern through the demarcated (white rectangle) region of the sarcolemma of this representative section.

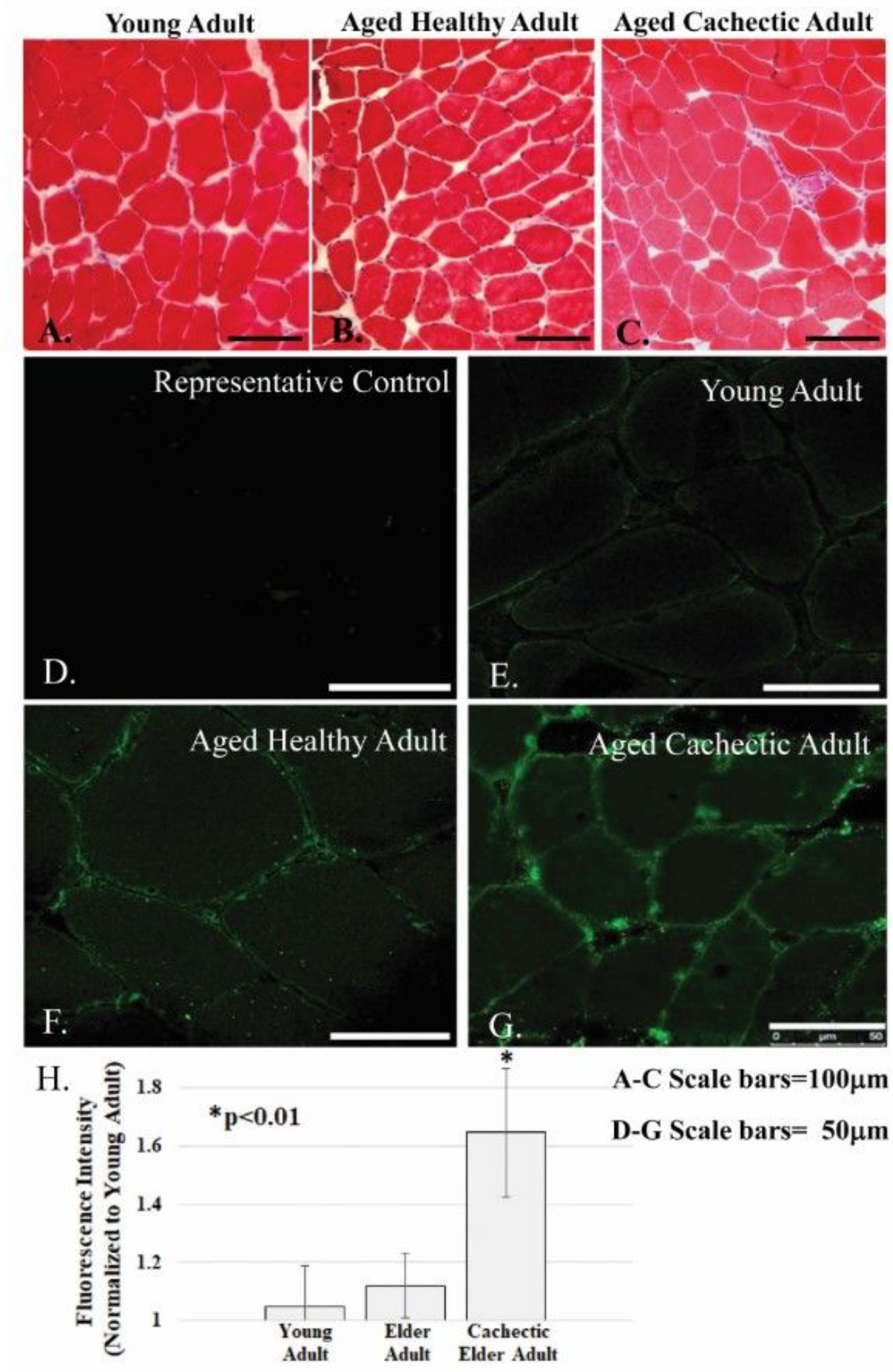

Figure 2 
ERG1 protein was detected in cross sections of human Rectus abdominis muscle by fluorescent immunohistochemistry. A-C. RA muscle sections stained with hemotoxylin and eosin demonstrate that muscle structure and diameter are normal in the samples from the healthy (young and aged; $A$ and $B$, respectively) people while muscle from the cachectic aged persons (C) had decreased myofiber diameter. Scale bars A-C are $100 \mathrm{Dm}$. D. Fluorescence was absent from sections immuno-stained without primary antibody. E-G. The ERG1 fluorescence was greater in the cachectic skeletal muscle of aged humans (G, $n=5)$ than in that of non-cachectic aged-matched $(F, n=7)$ or young adult $(E, n=4)$ humans. All samples were stained and then imaged using the same parameters in a single setting. Scale bars $D-G$ are 50 um. E. Graph bars represent mean fluorescence intensity \pm the standard error of the mean. 


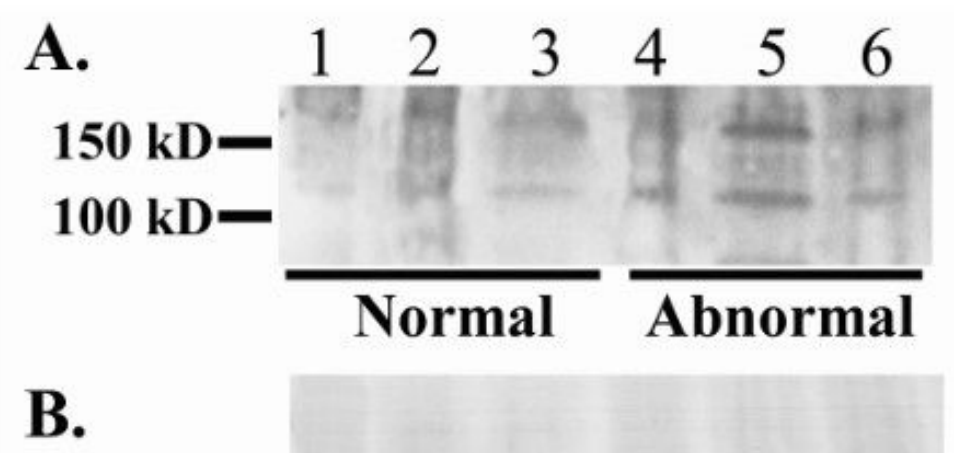

\section{$50 \mathrm{kD}$ - \\ 37 kD-}

C.
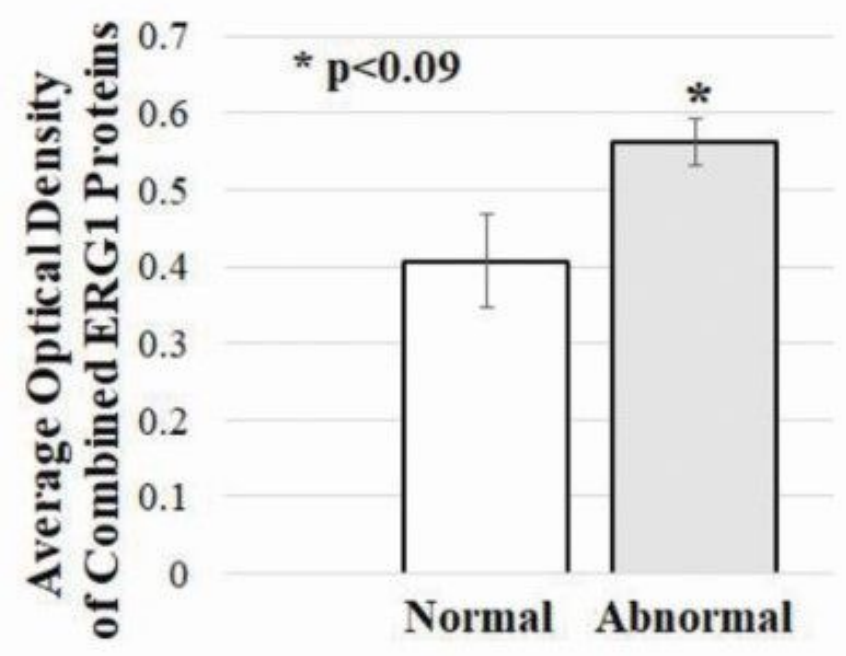

Normal Abnormal

D.

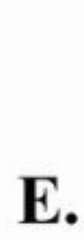

$150 \mathrm{kD}$

100 kD-

75 kD-

$50 \mathrm{kD}-$

37 kD -

\section{Figure 3}

ERG1a protein glycosylated variants are more abundant in skeletal muscle of cachectic than healthy humans. A. Human limb skeletal muscle membrane homogenates were prepared from both cachectic (Abnormal) and healthy (Normal) humans and immunoblotted $(50 \mathrm{gg})$ to show that the ERG1a protein is more abundant in the muscle from cachectic individuals. B. Coommassie stain of the immunoblotted membrane reveals that equal amounts of homogenate protein were loaded into the wells for SDS-PAGE. 
C. The graph demonstrates the difference in mean optical densities (OD) of the ERG1a proteins. The bars represent the average $\mathrm{OD} \pm$ the standard error of the mean. D,E. Immunoblot of Rectus abdominis muscle from normal (lane 1) and cachectic (lanes 2 and 3) humans suggests that the ERG1 protein may be more prominent in the muscle of cachectic people. The ERG1A protein is detected in the concentrated membrane proteins of malignant esophageal muscle (lane 4).

\section{Representative Control (red)}

\section{Representative Control (green)}

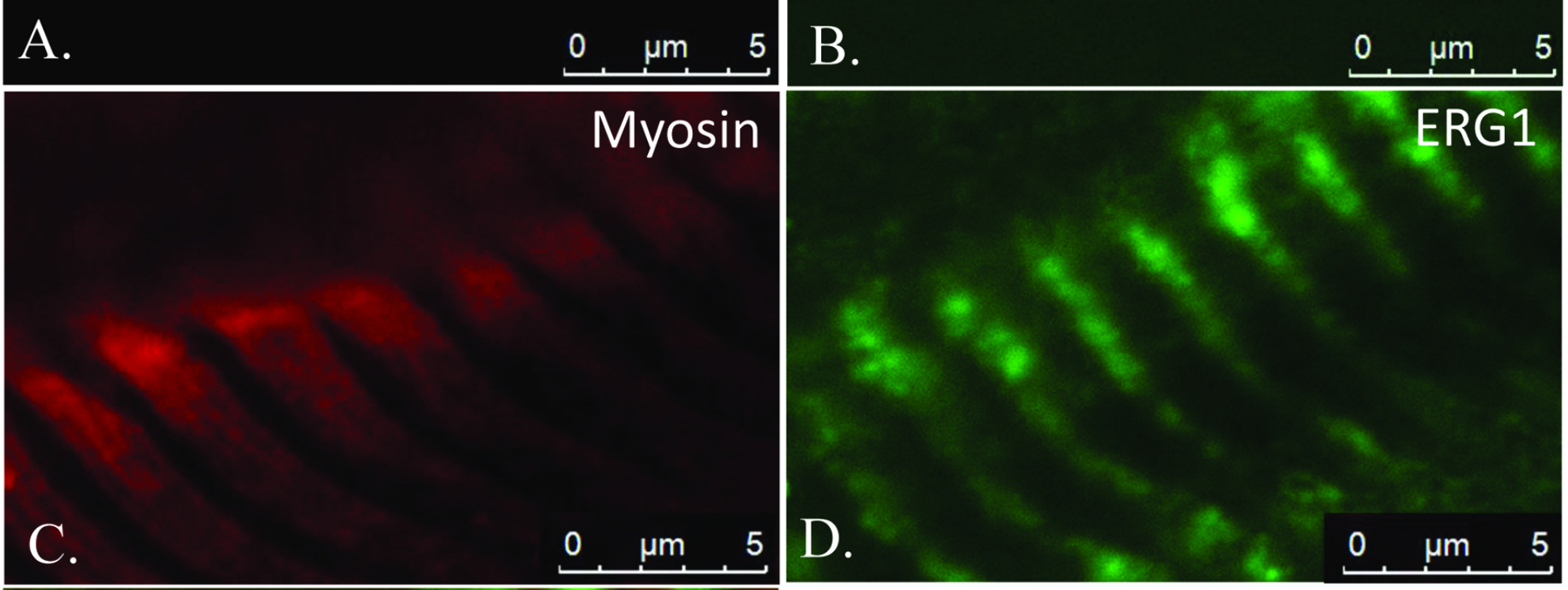

\section{Merged}

\section{Figure 4}

ERG1 (green) immunofluorescence localizes between bands of myosin (red) in cryo-sections of rat Gastrocnemius muscle. A (red), B (green). Fluorescence was absent from control older rat muscle 
sections immuno-stained without primary antibody. C. The myosin fluorescence (red) occurs in a "striped" pattern, with the red reflecting the A-band of the sarcomere while the non-fluorescent area (from which myosin is absent) connotes the I band. D. The ERG1 fluorescence (green) also occurs in a "striped" pattern with the fluorescent bands being narrower than those of the myosin. E. When merged, the ERG1 and myosin fluorescence do not co-localize, showing that there is no overlap of these proteins. Scale bars are $5 \square \mathrm{m}$ for all panels. F. Profile plot of multiple line scan data taken from a representative image of superimposed red and green fluorescence (white line in E) reveals that the highest fluorescence intensities for ERG1 (green) and myosin (red) areas do not significantly overlap with each other. 


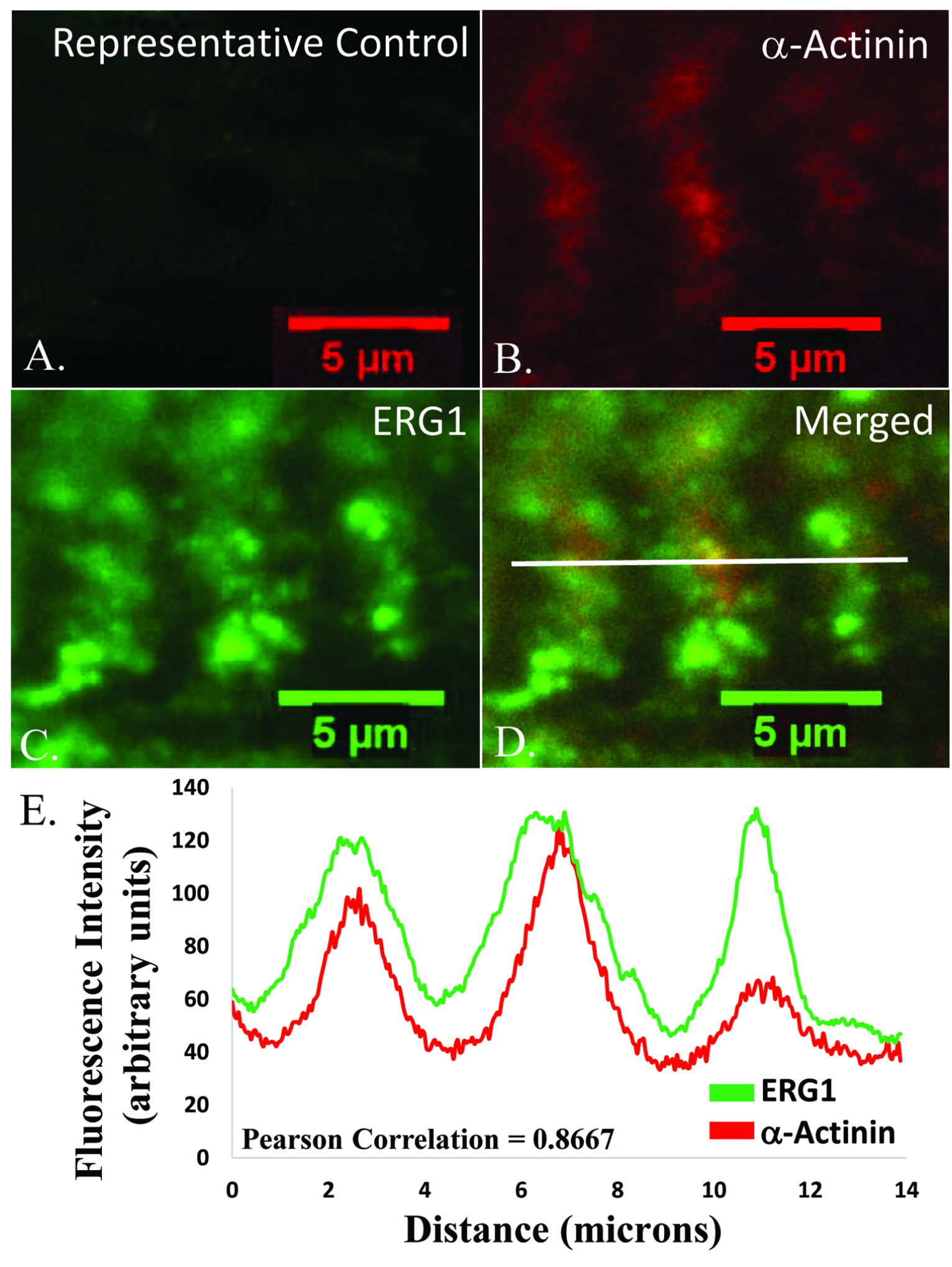

Figure 5

a-actinin fluorescence is detected within ERG1 fluorescence, suggesting ERG1 localizes within t-tubules in rat Gastrocnemius muscle. A. Fluorescence was absent from sections immuno-stained without primary antibody (representative section immunostained with 568 secondary antibody). B. \-Actinin immunofluorescence (red; a Z-line marker) was detected (as expected) in a pattern consistent with sarcomeric Z disks. C. ERG1 fluorescence (green) was also detected in a pattern consistent with t-tubule 
structure. D. When merged, 囚-actinin (red) immunofluorescence signal appears to fall within the ERG1 (green) immunofluorescent signal. Scale bars are $5 \square \mathrm{m}$ for all panels. E. ERG1 (green) and \-actinin (red) fluorescence intensities follow the same pattern through the demarcated (white line) region of the merged image (D), with the red falling within the green fluorescence as would be expected if the ERG1 were located in the t-tubules found on either side of the Z-line.

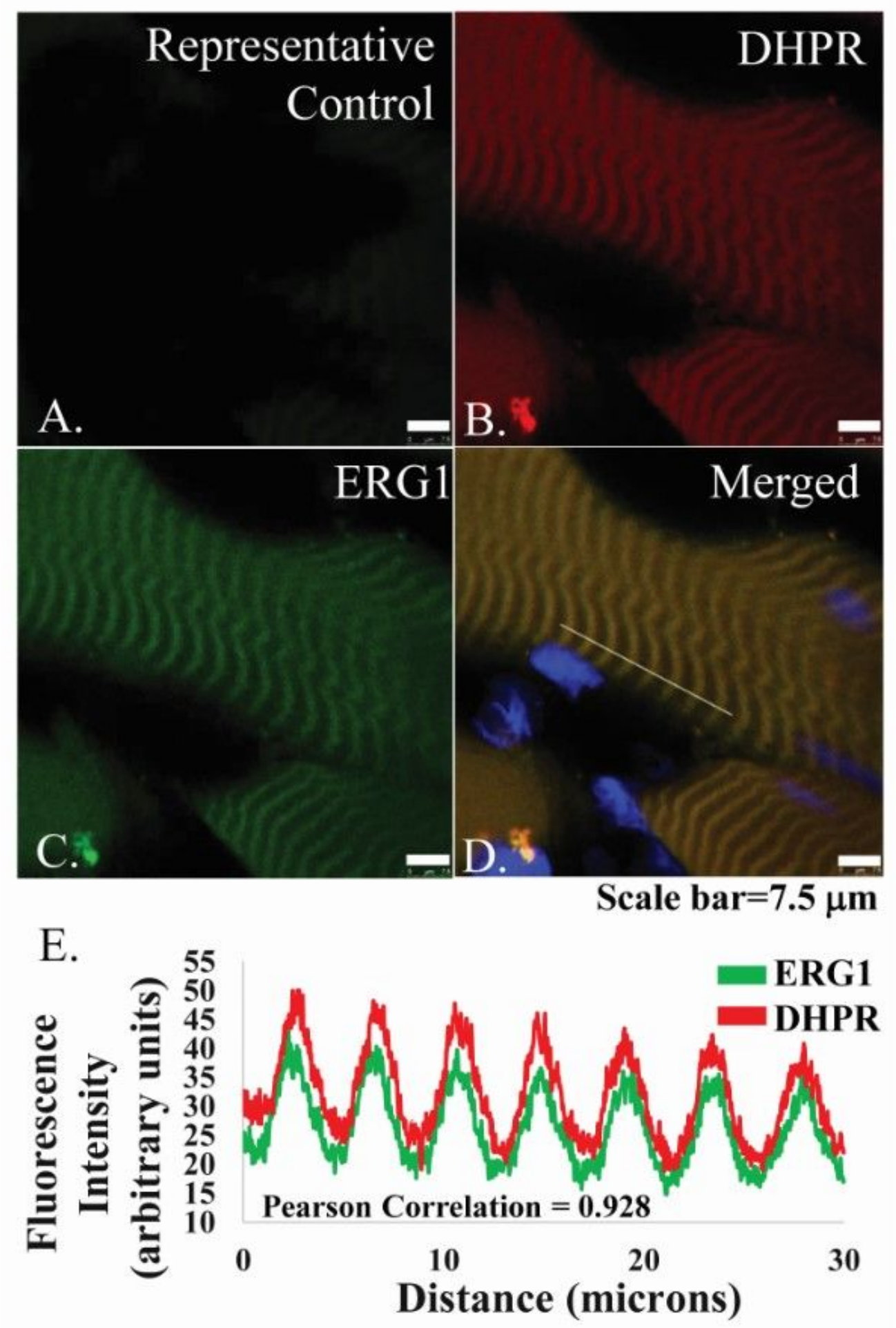

Figure 6 
ERG1 and dihydropyridine receptor (DHPR, a known t-tubule marker) immunofluorescences co-localize in rat Gastrocnemius muscle. A. Fluorescence was absent from muscle sections immuno-stained (red or green) without primary antibody. B. DHPR immunofluorescence (red) was detected (as expected) in a pattern consistent with muscle t-tubules. C. ERG1 fluorescence (green) was also detected in a pattern consistent with t-tubule structure. D. When merged, ERG1 (green) and DHPR (red) immunofluorescences appear to co-localize (yellow). Scale bars are $7.5 \square \mathrm{m}$ for all panels. E. ERG1 (green) and dystrophin (red) fluorescence intensities follow the same pattern through the demarcated (white line) region of the merged image (D). 